\title{
Clinical Characteristics and Evolution of Giant Cell Tumor Occurring in Paget's Disease of Bone
}

\author{
Domenico Rendina, ${ }^{1}$ Gianpaolo De Filippo, ${ }^{1}$ Stuart H Ralston, ${ }^{2}$ Daniela Merlotti, ${ }^{3}$ Fernando Gianfrancesco, ${ }^{4}$ \\ Teresa Esposito, ${ }^{4}$ Riccardo Muscariello, ${ }^{1}$ Ranuccio Nuti, ${ }^{3}$ Pasquale Strazzullo, ${ }^{1}$ and Luigi Gennari ${ }^{1}$ \\ ${ }^{1}$ Department of Medicine and Surgery, Federico II University, Naples, Italy \\ ${ }^{2}$ Rheumatic Diseases Unit, Molecular Medicine Centre, Western General Hospital, University of Edinburgh, Edinburgh, UK \\ ${ }^{3}$ Department of Medicine, Surgery and Neurosciences, University of Siena, Siena, Italy \\ ${ }^{4}$ Institute of Genetics and Biophysics "Adriano Buzzati-Traverso,", Italian National Research Council, Naples, Italy
}

\begin{abstract}
Patients with Paget's bone disease (PDB) have an increased risk of developing giant cell tumor (GCT). This study was performed to evaluate the clinical characteristics and evolution of GCT complicating PDB and to compare these clinical characteristics to those observed in two large PDB cohorts, the PDB Italian Registry and the United Kingdom's Multi-Centre Randomised Controlled Trial of Symptomatic Versus Intensive Bisphosphonate Therapy for Paget's Disease (PRISM) study. A systematic literature review identified 117 cases of PDB complicated by GCT (PDB-GCT), which involved the skeletal sites affected by PDB (110 patients) or the extraskeletal tissues adjacent to affected bones (7 patients). In contrast to what previously reported for GCT patients without GCT patients (83.2\%) were white and one-fourth of them (24.8\%) had multifocal GCTs. Compared to PDB patients without GCT, PDB-GCT patients showed a higher male/female ratio (2.1 versus 1.2) and more severe disease (age at PDB onset $52.1 \pm 12.1$ versus $63.3 \pm 10.6$ years; number of affected sites $6.1 \pm 2.9$ versus $2.34 \pm 1.6$; prevalence of polyostotic PDB 93.3\% versus $60.6 \%$ ). The mortality rate of PDB-GCT patients was higher than those occurring in GCT patients without PDB (about $50 \%$ versus $0 \%$ to $5 \%$ at 5 years) or in PDB patients without GCT (log rank =29.002). Moreover, up to $98 \%$ of PDB-GCT cases had elevated total alkaline phosphatase levels at neoplasm diagnosis, suggestive of active PDB. Importantly, PDB-GCT patients from Southern Italy (45.6\% of all GCT patients) showed a higher prevalence of multifocal GCT (51.7\%) and of positive familial history for PDB (70.8\%) and GCT (65.0\%). Finally, indirect evidence suggests a decline in the incidence of GCT in PDB patients. The occurrence of GCT in PDB patients is associated with severe disease and reduced life expectancy of affected patients. The increased prevalence of familial diseases in PDB-GCT patients from Southern Italy suggests a founder effect. The observed changes over time in the incidence of GCT in PDB patients could be related to improved clinical management and/or living conditions of patients. (c) 2014 American Society for Bone and Mineral Research.
\end{abstract}

KEY WORDS: PAGET'S BONE DISEASE; GIANT CELL TUMOR; SYSTEMATIC REVIEW; MORTALITY; INCIDENCE

\section{Introduction}

$\mathrm{P}$ aget's bone disease (PDB; OMIM\# 602080) is a focal disorder with a strong genetic component characterized by increased and disorganized bone remodeling, bone expansion, and abnormal bone structure. ${ }^{(1,2)}$ Among the several complications of this disorder, patients with PDB are at greatly increased risk of developing primary bone neoplasms as compared to controls. These mainly include osteosarcoma and, less frequently, giant cell tumor (GCT). ${ }^{(3-9)}$ Accounting for $6 \%$ of all primary bone neoplasms, GCTs are composed of multiple cell types. ${ }^{(10)}$ The name is based on the numerous multinucleated giant cells found within the tumor, which are responsible for the extensive local osteolysis. ${ }^{(10,11)}$ However, the neoplastic components of
GCT are the spindle-like stromal cells, which promote giant cell formation and largely direct the pathogenesis of the tumor. ${ }^{(10,12)}$ In contrast with osteosarcomas, although locally aggressive, GCTs rarely metastasize (2\% to $6 \%$ of cases). ${ }^{(13)}$ In a recent study in a large pedigree with GCTs complicating PDB (PDB-GCT patients), we demonstrated an increased disease severity associated with impaired response to antiresorptive treatment and an increased prevalence of cardiovascular complications. ${ }^{(14)}$ In order to confirm these findings and to enhance knowledge about this PDB complication, we performed a systematic review of all reported GCT cases in PDB patients and we compared disease characteristics of these patients with those observed in two large cohorts of unrelated PDB subjects.

Received in original form May 11, 2014; revised form August 17, 2014; accepted August 23, 2014. Accepted manuscript online September 4, 2014. Address correspondence to: Luigi Gennari, MD, PhD, Department of Medicine, Surgery and Neurosciences, University of Siena, viale Bracci 1, 53100 Siena, Italy. E-mail: gennari@unisi.it

Additional Supporting Information may be found in the online version of this article.

Journal of Bone and Mineral Research, Vol. 30, No. 2, February 2015, pp 257-263

DOI: 10.1002/jbmr.2349

(c) 2014 American Society for Bone and Mineral Research 


\section{Patients and Methods}

Data sources and searches

We conducted a systematic review to identify cases of GCT occurring in PDB patients (PROSPERO database registration number: CRD42014007030). The systematic search was performed according to Preferred Reporting Items for Systematic Reviews and Meta-Analyses (PRISMA) criteria (Supporting Fig. 1, Supporting Table 1). A total of 102 studies were included in the final synthesis (Supporting Table 2).

\section{Patient populations}

Clinical information from PDB-GCT cases were compared with those reported in two large cohorts of unrelated PDB patients from Italy and the UK, namely the Italian Registry of PDB $(n=718)$ and the United Kingdom's Multi-Centre Randomised Controlled Trial of Symptomatic Versus Intensive Bisphosphonate Therapy for Paget's Disease (PRISM) study $(n=774)$ (Table 1$)$.

\section{Statistical analysis}

At univariate analyses, statistical comparisons were based on the Student's $t$ test with Bonferroni correction when requested, for continuous variables, and on the $\chi^{2}$ test for dichotomous variables. Logistic regression models, based on the results of univariate analyses, were generated to evaluate the possible statistical interference between the given variables. Moreover, linear regression analysis and Pearson's correlation were performed in order to explore the degree of intervariable associations for interval and nominal variables, respectively. Kaplan-Meier survival curves were also obtained. Quantitative data were expressed as mean \pm SD. Statistical analysis was performed using a SPSS statistics package, version 11.5 (SPSS, Chicago, IL, USA) and Statistica $5 \cdot 1$ (Statsoft, Tulsa, OK, USA). All statistical tests were two-tailed. A $p$ value $<0.05$ was considered statistically significant.

\section{Results}

\section{Characteristics of GCT in PDB}

The systematic review identified 117 cases of PDB complicated by GCT (ie, PDB-GCT patients). In 110 patients (94\%) GCT occurred in bones affected by PDB (skeletal GCT), whereas in 7 cases (6\%) the neoplasm occurred in extraskeletal tissues (extraskeletal GCT), adjacent to bones affected by PDB (Supporting Table 3). PDB-GCT patients showed a higher male/ female ratio, an increased prevalence of polyostotic and familial PDB, and an earlier age at onset of PDB compared to PDB patients without GCT from the Italian Registry and the PRISM study (Table 1). The GCT was single in 88 cases and multifocal (synchronous or asynchronous) in 29 cases (Table 2). Twentyseven of the 110 patients with skeletal GCT (24.5\%) had multifocal GCT; among them, 18 had two GCTs and 9 had three or more GCTs. Up to 27 GCTs were described on more than one occasion in the same PDB patient in different reports. The mean age at onset of GCT was $62.5 \pm 11.7$ years. PDB patients with multifocal GCT showed a mean age at onset of the first neoplasm significantly lower compared to those with single GCT (Table 2). The large majority of PDB-GCT patients in whom race/ethnicity was reported $(65 / 79 ; 82.3 \%)$ were white (most of them of European ancestry), 8 (10.1\%) were black, and 6 (7.6\%) were Asian. No differences were observed in the prevalence of gender, single or multifocal GCT, and skeletal or extraskeletal GCT between racial/ethnic groups. Conversely, an earlier age at onset of GCT was observed in Asian PDB patients compared to white and black patients (Asian versus white versus black patients $=$ $48.8 \pm 9.3$ versus $62.4 \pm 10.2$ versus $62.2 \pm 13.7$ years; $p=0.011$ ). Fifteen out of 43 (35\%) PDB-GCT patients with available information had at least one first-degree relative affected by both diseases; in those 15 cases there was a higher prevalence of multifocal GCTs compared to patients without a familial history for PDB and GCT (9/15 versus 5/28). The mean age at onset of GCT in patients with familial PDB and GCT was lower but not significantly different compared to those observed in patients without familial PDB and GCT (56.8 \pm 6.1 versus $62.4 \pm 14.1$ years; $p=0.08$ ).

Details of the sites affected by skeletal GCT are also shown in Table 2. GCT involvement of the axial skeleton was observed in 78 cases (75\%). Interestingly, PDB patients with multiple skeletal GCT showed a preferential localization of neoplasms in the spine. Histological grading, according to criteria proposed by Jaffe, ${ }^{(15)}$ was available in 101 cases. Overall, GCT lesions of grade 1, 2, and 3 were observed in 25 (24.7\%), 52 (51.5\%), and $24(23.8 \%)$ patients, respectively. Histological specimens of 13 PDB-GCT cases since 1981 were also examined to evaluate the presence of intracellular viral-like inclusions, which were documented in $11(84.6 \%)$ cases.

Table 1. Clinical Characteristics of PDB Patients With or Without GCT

\begin{tabular}{lcccc}
\hline & Overall PDB-GCT & White PDB-GCT & Italian PDB Registry & PRISM Study (UK) \\
\hline Patients, $n$ & 117 & 65 & 718 & 765 \\
Gender (male versus female), $\%$ & 67.6 versus $32.4^{\mathrm{a}}$ & 66.2 versus $33.8^{\mathrm{a}}$ & 57.0 versus 43.0 & $53.2 \mathrm{versus} 46.8$ \\
Polyostotic PDB, $\%$ & $93.3^{\mathrm{b}}$ & $90.8^{\mathrm{b}}$ & 58.9 & 62.3 \\
Affected PDB sites, $n$ & $6.1 \pm 2.9^{\mathrm{c}}$ & $6.6 \pm 2.9^{\mathrm{c}}$ & $2.5 \pm 1.9$ & $2.2 \pm 1.2$ \\
Positive family history of PDB, $\%$ & $58.5^{\mathrm{d}}$ & $62.9^{\mathrm{e}}$ & 32.1 & 12.7 \\
Age of PDB onset, years & $52.1 \pm 12.1^{\mathrm{f}}$ & $52.4 \pm 11.2^{\mathrm{g}}$ & $59.3 \pm 11.9$ & $67.0 \pm 9.8$ \\
\hline
\end{tabular}

$\mathrm{PDB}=$ Paget's disease of bone; $\mathrm{GCT}=$ giant cell tumor.

${ }^{\mathrm{a}}$ Data available in 111 PDB-GCT patients; $p=0.038$ versus Italian PDB patients and $p=0.006$ versus PRISM PDB patients.

${ }^{\mathrm{b}}$ Data available in 74 PDB-GCT patients; $p<0.0009$ versus Italian PDB patients and versus PRISM PDB patients.

${ }^{c}$ Data available in 74 PDB-GCT patients; $p=0.001$ versus Italian PDB patients and $p<0.0009$ versus PRISM PDB patients.

${ }^{\mathrm{d}}$ Data available in 41 PDB-GCT patients; $p=0.001$ versus Italian PDB patients and $p<0.0009$ versus PRISM PDB patients.

$\mathrm{e}_{p}<0.0009$ versus PRISM PDB patients and versus Italian PDB patients.

fData available in 92 PDB-GCT patients; $p=0.031$ versus Italian PDB patients and $p<0.0009$ versus PRISM PDB patients.

${ }^{9} p=0.038$ versus Italian PDB patients and $p=0.001$ versus PRISM PDB patients. 
Table 2. Clinical Characteristics of PDB-GCT Patients With Single or Multifocal GCT

\begin{tabular}{|c|c|c|c|}
\hline & Single GCT & Multifocal GCT & Total \\
\hline Patients, $n$ (\%) & $88(76.2)$ & $29(24.8)$ & 117 \\
\hline Skeletal:extraskeletal GCT, $n$ & 83:5 & $27: 2$ & $110: 7$ \\
\hline Gender (male:female), $n$ & $56: 27$ & 19:9 & 75:36 \\
\hline Age at onset of GCT, years (mean $\pm \mathrm{SD}$ ) & $64.2 \pm 12.2$ & $57.4 \pm 8.6^{\mathrm{a}}$ & $62.5 \pm 11.7$ \\
\hline Ethnicity (white:black:Asian), $n$ & 47:7:3 & 18:1:3 & 65:8:6 \\
\hline Family history of PDB-GCT (yes:no), $n$ & $6: 23$ & $9: 5^{b}$ & $15: 28$ \\
\hline ALP serum levels at GCT onset, $\%^{\mathrm{b}}$ & $775 \pm 632$ & $1020 \pm 667$ & $834 \pm 644$ \\
\hline GCT involving axial skeleton, $n$ & 55 & 23 & 78 \\
\hline GCT involving skull and mandible & 43 & 12 & 55 \\
\hline GCT involving pelvis & 12 & 7 & 19 \\
\hline GCT involving spine & 8 & $10^{d}$ & 18 \\
\hline GCT involving symmetrical bones, $n$ & 16 & 10 & 26 \\
\hline Age at onset of $\mathrm{PDB}$, years (mean $\pm \mathrm{SD}$ ) & $53.5 \pm 12.4$ & $48.2 \pm 10.4$ & $52.1 \pm 12.1$ \\
\hline Time between PDB and GCT diagnoses, years (mean \pm SD) & $12.9 \pm 8.7$ & $11.5 \pm 9.7$ & $12.5 \pm 8.9$ \\
\hline Family history of PDB (yes:no), $n$ & 13:5 & 11:12 & $24: 17$ \\
\hline Skeletal sites involved by PDB, $n$ (mean \pm SD) & $5.9 \pm 3.1$ & $6.3 \pm 2 \cdot 5$ & 6.1 \\
\hline
\end{tabular}

Values were determined from PDB-GCT patients with available information for each characteristic.

$\mathrm{ALP}=$ alkaline phosphatase; PDB = Paget's disease of bone; GCT = giant cell tumor; PDB-GCT = PDB patients with GCT.

${ }^{a}$ Significantly different compared to PDB patients with single GCT, $p=0.009$.

${ }^{\mathrm{b}}$ Significantly different compared to PDB patients with single GCT, $p=0.008$.

'The mean levels of ALP at the diagnosis of GCT are expressed as percentages compared to the maximum laboratory reference range.

dSignificantly different compared to PDB patients with single GCT, $p=0.003$.

\section{Characteristics of PDB in PDB-GCT patients}

Data regarding the extent of PDB were available in 104 PDB-GCT patients. The mean number of skeletal sites affected by PDB in the 97 PDB-GCT patients with polyostotic disease was $6.1 \pm 2.9$ sites (range, 2 to 14 sites), and there were no major differences between cases with single or multifocal GCT (Table 2). The axial skeleton and the symmetrical bones were involved in at least 87 $(89.7 \%)$ and $66(68.0 \%)$ of polyostotic PDB cases, respectively. Among the 7 patients with monostotic PDB, the skull and the mandible were affected in 2 patients, whereas the femur, tibia, and pelvis were described in 1 patient each. The mean age at onset of PDB did not differ in relation to gender, family history for PDB, or between extraskeletal or skeletal GCTs. Conversely, a trend for an earlier age of onset of PDB was observed in multifocal versus single GCT cases $(48.2 \pm 10.4$ versus $53.3 \pm 12.4$ years, respectively; $p=0.06$ ). Moreover, the age at onset of PDB was significantly lower in Asian patients compared to white and black patients (Asian versus white versus black patients: $38.6 \pm 12.7$ versus $52.4 \pm 11.2$ versus $52.0 \pm 15.7$ years; $p=0.036$ ). Information concerning the time frame between the age at onset of PDB and the occurrence of GCT was available in 91 patients; in 21 patients $(23.1 \%)$ the diagnosis of PDB and GCT were made at the same time, whereas in the remaining 70 cases $(76.9 \%)$ the diagnosis of PDB preceded the occurrence of GCT by $12.5 \pm 8.9$ years (range, 1 to 39 years). Interestingly, in these latter patients a direct relationship was observed between the ages at onset of PDB and GCT ( $r=0.703, p=0.001$; Supporting Fig. 2). The time frame between the diagnosis of PDB and CGT was similar in those with single or multifocal GCT (Table 2).

Overall, 24 of 41 PDB-GCT cases with available information (58.5\%) had at least one first-degree relative affected by PDB. In patients with familial PDB, the age at onset of PDB, the number of skeletal sites, the age at onset of GCT, and the prevalence of single and multifocal GCT were not significantly different compared to GCT cases without familial PDB. Analyzing the decade at onset of GCT complicating PDB, a progressive decrease is evident in the percentage of white PDB-GCT cases (either Italian or other non-Italian whites) diagnosed during the last 40 years, with only few cases diagnosed after 1990. An opposite trend was observed with respect to the decade at onset of PDB cases without GCT from the Italian Registry and the PRISM study cohort (Fig. 1, Supporting Fig. 3). This indirectly suggests a decrease in the prevalence of GCT with age in PDB patients.

\section{Clinical signs and course of GCT}

\section{Clinical signs of GCT}

The clinical signs and symptoms at the onset of GCT were available for 99 PDB-GCT patients. The most frequent clinical sign associated with GCT occurrence was the deformity of bones or

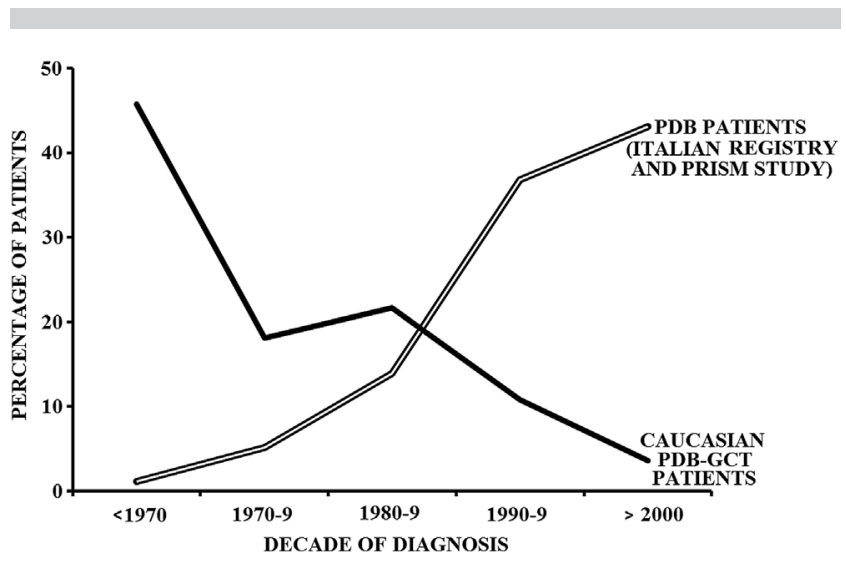

Fig. 1. Changes over time in the prevalence of GCT occurring in white (Italian and other non-Italian) patients with PDB and of PDB diagnosis in white PDB patients (without personal and familial history of GCT) from the Italian PDB Registry and the PRISM study cohort. GCT = giant cell tumor; $\mathrm{PDB}=$ Paget's disease of bone. 
extraskeletal tissues involved by the neoplasm(s). This sign was observed in 72 PDB-GCT patients (72.7\%), of whom 69 had skeletal GCT: 49 of 54 patients with GCT of the skull and the mandible (90.7\%); 6 of 10 patients with GCT of the long bones $(60.0 \%) ; 10$ of 19 patients with GCT of the pelvis (52.6\%); and in 4 of 11 PDB-GCT patients with GCT of the spine (36.4\%). While the count of affected skeletal sites may overlap, the number of GCT cases with deformity was 72, 69 with bone deformity and 3 with deformity at extraskeletal sites $(69+3=72)$. The second most frequent clinical feature of GCT was pain, which was referred by 50 patients $(50.5 \%$, including 4 with extraskeletal GCT). This symptom was reported by $75 \%$ of patients with GCT of the axial skeleton. In this setting, pain was linked to physical compression of neighboring nerves rather than to bone pain. Bleeding was observed in $10 \mathrm{GCT}$ cases (owing to neoplastic involvement of the splanchnocranium in 9 cases, and to bladder involvement of a pelvic GCT in 1 case). Conversely, in the 4 PDB-GCT cases with GCT involving symmetrical bones, the clinical diagnosis of GCT was related to the occurrence of a fracture. Serum levels of total alkaline phosphatase (ALP) at the onset of GCT were reported in 71 patients. Only 1 patient showed ALP levels within the normal range, whereas the remaining 70 patients (98.6\%) showed elevated ALP levels. The mean levels of ALP at the diagnosis of $G C T$, expressed as percentages compared to the maximum laboratory reference range, were not significantly different between PDB patients with single or multifocal GCT (Table 2).

\section{Management and clinical course of GCT}

Data regarding the treatment of GCT are summarized in Supporting Table 4. The therapeutic approach to GCT was influenced by: (1) the decade at onset of neoplasm (medical therapy was considered a viable therapeutic alternative after the 1970s); (2) the GCT histological grade (surgical therapy alone or in combination was preferred in GCT grades 1 [20/23] and 2 [35/44], whereas radiotherapy alone or in combination was preferred in GCT grade 3 [13/21]); (3) the clinical signs and symptoms at the onset of GCT (those with the compression of neighboring structures [13/20] and all cases with a bone fracture were preferentially managed with a surgical approach alone or in combination); (4) the characteristics of GCT (multiple GCT [17/26] and extraskeletal GCT [4/5] were preferentially treated by surgery alone or in combination); and (5) the skeletal site affected by GCT (GCT involving the skull [40/52], the spine [10/14], and symmetrical long bones [10/14] were preferentially treated by surgery alone or in combination; GCT involving the pelvis [10/15] was treated preferentially by radiotherapy alone or in combination). Information regarding the clinical course of GCT was available in 94 patients. The local recurrence of GCT was reported in 26 cases and occurred between 1 and 36 years, with a mean lapse of time of $7.9 \pm 10.7$ years. No significant differences were found between patients with or without local recurrence of GCT with respect to the age at onset of GCT, the percentage ALP levels at the onset of GCT, the number of skeletal sites affected by PDB, and the prevalence of familial or sporadic PDB (Supporting Table 5).

At the end of the observation time, 36 PDB-GCT patients were dead (mean age at death $67.9 \pm 11.2$ years). The clinical characteristics of PDB-GCT patients with a fatal outcome, compared with those who were alive at the end of the observation time are reported in Table 3 ; the survival curve from GCT diagnosis is shown in Fig. $2 A$. Of interest, the PDB-GCT patients who died at the end of the observation time were older, showed higher ALP levels, and had a higher prevalence of histological grade $3 \mathrm{GCT}$ at onset of GCT compared to those still alive. A Cox regression analysis confirmed that age, ALP levels, and histological grade at GCT onset significantly predict the prognosis with regard to survival of PDB-GCT patients. Moreover, PDB-GCT patients showed a survival curve from PDB diagnosis significantly different compared to PDB patients without GCT (log rank $=29.002 ; p=0.001$; Fig. $2 B$ ). The causes of death that were reported for these GCT cases are summarized in Supporting Table 6. Of interest, at least 21 PDB-GCT patients reported the occurrence of cardiovascular disorders. A positive family history for PDB and/or GCT was evidenced in at least 8 of these 21 patients and most of these familial cases were from Campania, in Southern Italy.

\section{Characteristics in PDB-GCT cases of Italian descent}

An increased prevalence of subjects form Italy or with Italian ancestors was observed in PDB-GCT patients described to date. At least 36 of 79 PDB-GCT patients of known nationality $(45.6 \%$, 23 males and 13 females) were Italian or with Italian ancestors; among these 36 patients, 29 (18 males and 11 females) were from Avellino or neighboring cities of Naples and Foggia. The age at onset of GCT in these Italian patients was no different compared to the other white patients or to the other racial/

Table 3. Clinical Characteristics of PDB-GCT Patients Dead or Alive at the End of Observation Time

\begin{tabular}{|c|c|c|c|}
\hline & Dead & Alive & Total \\
\hline Patients, $n(\%)$ & $36(38.3)$ & $58(41.7)$ & 94 \\
\hline Observation time, years & $3.9 \pm 5.5$ & $4.1 \pm 3.4$ & $4.0 \pm 4.4$ \\
\hline Single: multifocal GCT, $n$ & $27: 9$ & $41: 17$ & $68: 26$ \\
\hline $\begin{array}{l}\text { Recurrent:nonrecurrent } \\
\text { GCT, } n\end{array}$ & $6: 23$ & $20: 35$ & $26: 58$ \\
\hline $\begin{array}{l}\text { Skeletal:extraskeletal } \\
\text { GCT, } n\end{array}$ & $35: 1$ & $50: 5$ & $85: 6$ \\
\hline Gender (male:female), $n$ & $22: 13$ & $36: 17$ & $58: 30$ \\
\hline $\begin{array}{l}\text { Age at onset of GCT, } \\
\text { years }\end{array}$ & $64.3 \pm 11.6^{\mathrm{a}}$ & $59.7 \pm 9.8$ & $61.5 \pm 11.5$ \\
\hline $\begin{array}{l}\text { ALP serum levels } \\
\text { at GCT onset, } \%^{b}\end{array}$ & $1248 \pm 790^{c}$ & $652 \pm 528$ & $841 \pm 683$ \\
\hline $\begin{array}{l}\text { Histological grade } \\
\qquad(1: 2: 3), n\end{array}$ & $5: 12: 17^{d}$ & $15: 31: 6$ & $20: 43: 23$ \\
\hline $\begin{array}{l}\text { Family history of } \\
\text { PDB-GCT (yes:no), } n\end{array}$ & $5: 9$ & $7: 17$ & $12: 26$ \\
\hline $\begin{array}{l}\text { Age at onset of PDB, } \\
\text { years }\end{array}$ & $51.6 \pm 12.6$ & $51.5 \pm 11.2$ & $51.5 \pm 11.6$ \\
\hline $\begin{array}{l}\text { Long bones involved } \\
\text { by GCT, } n\end{array}$ & 5 & 8 & 13 \\
\hline $\begin{array}{l}\text { Axial skeleton involved } \\
\text { by } \mathrm{GCT}, n\end{array}$ & 6 & 27 & 33 \\
\hline $\begin{array}{l}\text { Family history of PDB } \\
\text { (yes:no), } n\end{array}$ & 7:5 & $12: 12$ & 19:17 \\
\hline $\begin{array}{l}\text { Skeletal sites involved by } \\
\text { PDB, } n \text { (mean } \pm \text { SD) }\end{array}$ & $6.5 \pm 3.7$ & $6.2 \pm 2.7$ & $6.3 \pm 3.1$ \\
\hline
\end{tabular}

$\mathrm{ALP}=$ alkaline phosphatase; $\mathrm{PDB}=$ Paget's disease of bone; $\mathrm{GCT}=$ giant cell tumor.

${ }^{\mathrm{a}} p=0.021$.

${ }^{\mathrm{b}}$ The mean levels of ALP at the diagnosis of GCT are expressed as percentages compared to the maximum laboratory reference range.

${ }^{c} p=0.003$.

${ }^{\mathrm{d}} p=0.01$. 

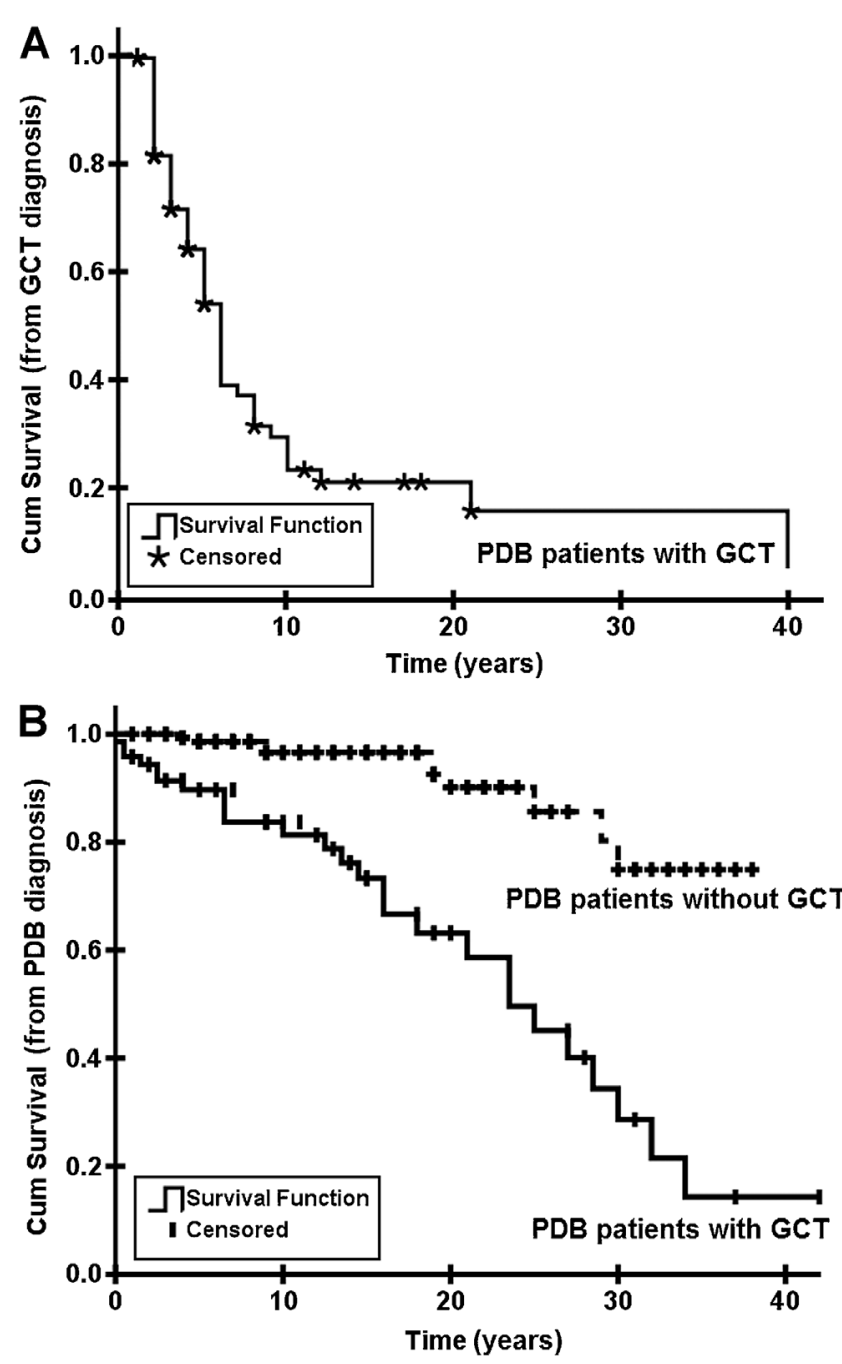

Fig. 2. (A) Survival curve of patients with PDB complicated by GCT from time of GCT diagnosis. (B) Survival curves of PDB patients with and without GCT from time of PDB diagnosis. GCT = giant cell tumor; $\mathrm{PDB}=$ Paget's disease of bone.

ethnic groups. However, at least 15 PDB patients with multifocal GCT (51.7\%, 8 males and 7 females) were Italian and 13 of them (7 males and 6 females) were from Avellino or neighboring cities. Thus, the prevalence of multifocal GCT was highest in patients from that Italian area. Moreover, the large majority of familial PDB cases with GCT were Italian (21/24, 87.5\%; $p=0.009)$, and all these familial Italian cases were from Avellino and neighboring cities. Comparing the clinical characteristics of PDB-GCT cases from Avellino and neighboring cities to those observed in 236 PDB patients (without familial or personal history of GCT) from that same geographical area, we observed that PDB-GCT patients had a lower age at onset of PDB, a higher number of affected skeletal sites, an increased prevalence of familial PDB, and a lower prevalence of PDB involvement of the appendicular skeleton (Table 4).

\section{Discussion}

In this study we have for the first time conducted a systematic review of the clinical characteristics and the evolution of GCT
Table 4. Clinical Characteristics of PDB Patients With or Without GCT From Avellino and Neighboring Cities

\begin{tabular}{|c|c|c|}
\hline & PDB-GCT ${ }^{a}$ & $\begin{array}{l}\text { Italian PDB Registry } \\
\text { (Campania cohort) }^{\mathrm{b}}\end{array}$ \\
\hline Patients, $n$ & 29 & 236 \\
\hline $\begin{array}{l}\text { Gender (male versus } \\
\text { female), } \%\end{array}$ & 62.1 versus 37.9 & 58.2 versus 41.8 \\
\hline Polyostotic PDB, \% & 93.3 & 72.8 \\
\hline Affected PDB sites, $n$ & $6.4 \pm 2.6^{c}$ & $2.8 \pm 2.0$ \\
\hline $\begin{array}{l}\text { Positive family history } \\
\text { of PDB, } \%\end{array}$ & $70.8^{\mathrm{d}}$ & 20.8 \\
\hline Age of PDB onset, years & $50.1 \pm 7.4^{\mathrm{e}}$ & $58.9 \pm 6.1$ \\
\hline $\begin{array}{l}\text { Involvement of } \\
\text { appendicular } \\
\text { skeleton, } \%\end{array}$ & $20.8^{\mathrm{d}}$ & 62.7 \\
\hline $\begin{array}{l}\text { Involvement of axial } \\
\text { skeleton, \% }\end{array}$ & 91.7 & 82.6 \\
\hline Age of GCT onset, years & $57.6 \pm 5.9$ & - \\
\hline $\begin{array}{l}\text { Positive family history } \\
\text { of GCT, \% }\end{array}$ & 65.0 & - \\
\hline $\begin{array}{l}\text { GCT type (single versus } \\
\text { multifocal), } \%\end{array}$ & 56 versus 44 & - \\
\hline
\end{tabular}

$\mathrm{PDB}=$ Paget's disease of bone; $\mathrm{GCT}=$ giant cell tumor.

${ }^{\mathrm{a} P D B}-\mathrm{GCT}$ cases from Avellino and neighboring cities.

${ }^{\mathrm{b}} \mathrm{PDB}$ patients from Avellino and neighboring cities, selected from the Italian PDB Registry, without personal and/or familial history of GCT.

'Significantly different compared to PDB patients from Campania without GCT, $p=0.002$.

${ }^{\mathrm{d}}$ Significantly different compared to PDB patients from Campania without GCT, $p<0.0009$.

esignificantly different compared to PDB patients from Campania without GCT, $p=0.011$.

occurring in PDB. Our analysis indicates that this neoplasm involves skeletal sites affected by PDB or extraskeletal tissues adjacent to them. Moreover, GCT occurring in PDB patients shows several peculiar characteristics compared to GCT occurring in absence of PDB (Table 5). In fact, GCT unrelated to PDB is usually a neoplasm growing eccentrically in the epi-metaphysis of long bones and in nearly $50 \%$ of cases affects the knee. ${ }^{(16-18)}$ Conversely, in PDB patients, GCT preferentially involves the axial skeleton and in particular the skull and facial bones. ${ }^{(19)}$ Furthermore, GCT unrelated to PDB occurs preferentially in females, with a male/female ratio of $1 / 1.5{ }^{(16)}$ whereas $75 \%$ of PDB-GCT patients were males. Although up $25 \%$ of PDB-GCT

Table 5. Comparison Between GCTs Occurring in Non-PDB and PDB Patients

\begin{tabular}{lcc}
\hline & Non-PDB GCT & PDB-GCT \\
\hline $\begin{array}{l}\text { Gender } \\
\text { Preferential race/ } \\
\text { ethnicity }\end{array}$ & female $>$ male & male $>$ female \\
Age of onset, years & $20-40$ & White \\
$\begin{array}{l}\text { Preferentially affected } \\
\text { skeletal sites }\end{array}$ & Appendicular bone & Axial bone \\
Multifocal GCT, \% & $<1$ & 25 \\
5-Year survival rate, \% & $96-100$ & $<50$ \\
\hline
\end{tabular}

$\mathrm{GCT}=$ giant cell tumor; $\mathrm{PDB}=$ Paget's disease of bone. 
patients developed multiple neoplasm, multifocal GCT unrelated to PDB is a very rare entity that occurs in less than $1 \%$ of cases. ${ }^{(16,20,21)}$ Also, the ethnicity and the age at onset are remarkably different: GCT unrelated to PDB is more common in China and India, where it constitutes approximately $20 \%$ of all bone tumors ${ }^{(16)}$; PDB-related GCT occurs preferentially in white subjects of European ancestry. In patients without PDB, GCT occurs in young adults, most often between 20 and 40 years of age, ${ }^{(16-18,22)}$ whereas pagetic GCT generally occurs after the fourth decade of life. Of interest, PDB-GCT patients had generally elevated serum ALP levels at GCT onset even though many had previously received pharmacological treatment for PDB, whereas in adult patients with GCT alone (without PDB) ALP levels where not different compared to controls. ${ }^{(23)}$ This observation suggests that GCT generally complicates a longstanding, active, and polyostotic PDB and is consistent with our previous remarks. ${ }^{(14)}$ One cannot, however, exclude that high ALP levels are caused by GCT directly eroding bone. Importantly, the analysis of the cumulative survival curve indicates that about $80 \%$ of PDB patients died within 10 years from the GCT clinical occurrence and $50 \%$ within 5 years. This 5 -year survival rate is significantly lower than that described for nonmalignant GCT occurring in the absence of PDB (from $96 \%$ to $100 \%$ ). ${ }^{(16)}$ Moreover, when the time of PDB diagnosis was considered, a consistent increase in mortality was observed in PDB-GCT cases compared to PDB cases without GCT degeneration. The older age, higher ALP levels at onset of GCT, and the GCT histological grading were the factors that significantly and independently influenced the survival of PDB-GCT patients. Death from cardiovascular events was also common, particularly in familial cases and in patients whose ancestors were from the Italian region of Campania, in keeping with our previous observations. ${ }^{(14)}$ Osteosarcoma is another rare but deadly neoplastic complication of PDB. Indeed, some clinical characteristics of GCT and osteosarcoma complicating PDB are similar, such as the skeletal localization and the preferential occurrence in males after the fourth decade of age. ${ }^{(6-9)}$ Nevertheless, contrary to GCT, multifocal osteosarcomas were rarely described in PDB patients. Interestingly, even though the occurrence of osteosarcomas was described in 3 of 33 (9.1\%) and 2 of $70(2.8 \%)$ patients with familial PDB in two separate studies, ${ }^{(24,25)}$ the presence of osteosarcoma in multiple affected members of the same family was reported only in 2 Italian siblings from Campania with long-standing and polyostotic PDB. ${ }^{(25)}$

Available data also demonstrate a significant prevalence of familial GCT complicating PDB in Southern Italy (Campania region and neighboring areas). This geographical area is historically a unique macroregion ${ }^{(26)}$ and is characterized by an increased clinical severity of PDB in both sporadic and familial patients. ${ }^{(27,28)}$ Consistent with this observation, the PDB-GCT patients from Campania and neighboring areas showed a more severe clinical phenotype in terms of age at onset of PDB, number of skeletal sites affected by PDB, ALP serum levels at PDB diagnosis, and prevalence of familial PDB disease, compared to those observed in the two cohorts of PDB cases from Italy and the United Kingdom and even in the subgroup of PDB patients from the same geographic area without personal and familial history of GCT. In particular, a consistent proportion of GCTs occurring in this Italian region had a positive family history and was more likely to be multifocal with compared to GCT occurring in other white patients from different countries or in other racial/ethnic groups. Thus, at least in these familial cases from Italy, the pathogenesis of GCT could be related to both genetic and environmental factors. Indeed, the GCT degeneration also occurs in PDB patients with ancestors in Southern Italy but who were born and live in different continents (ie, North and South America). This phenomenon could be justified by a founder effect with genetic drift or selection. ${ }^{(29)}$ Current evidence suggests that PDB is caused by a combination of rare alleles of large effect size that cause autosomal dominant inheritance of the disease and more common alleles of smaller effect size. ${ }^{(30-32)}$ Importantly, all PDB patients with GCT and their first-degree relatives so far examined were negative for the presence of SQSTM1 mutations. ${ }^{(14,28)}$ Moreover, a recent genomewide linkage analysis identified six possible candidate regions on chromosomes 1, 5, 6, 8, 10, and 20. ${ }^{(14)}$ On the other hand, the analysis of the changes over time in the prevalence of GCT in PDB patients from Campania suggests a decrease in the prevalence of this complication not related to a parallel decrease in the prevalence of PDB diagnosis. A similar conclusion was also reached after analysis of the age of diagnosis of other white GCTPDB patients compared with either the Italian Registry or the PRISM study cohorts of PDB patients without GCT (no GCT cases were reported in these populations). One can argue that the improved environmental conditions in most geographical areas and the recent progress in the pharmacological management of PDB (with the introduction of more potent intravenous bisphosphonate regimens) could have reduced the occurrence of this complication, as has also been reported for osteosarcoma complicating PDB. ${ }^{(5)}$

In conclusion, our analysis provided novel information concerning the characteristics and the clinical course of GCT complicating PDB that can be relevant in clinical practice for both the prevention and the management of this rare but serious complication. Additional studies are needed to dissect the genetic defects associated with the occurrence of GCT in SQSTM1-negative pedigrees.

\section{Disclosures}

All authors state that they have no conflicts of interest.

\section{Acknowledgments}

This work was supported by a grant from Telethon-Italy (grant 11119A); we gratefully acknowledge the financial support. We are grateful to all members of the PRISM trial group (the complete list is provided in the Supporting Information Acknowledgments).

Authors' roles: Study design: DR, GDF, and LG. Study conduct: DR, GDF, LG, and DM. Data collection and analysis: DR, LG, DM, RM, TE, FG, GDF, and SHR. Drafting manuscript: DR, GDF, SHR, and LG. Revising manuscript content: DR, LG, SHR, RN, and PS.

\section{References}

1. Ralston SH. Clinical practice. Paget's disease of bone. N Engl J Med. 2013:368:644-50.

2. Singer FR, Mills BG, Gruber HE, Windle JJ, Roodman GD. Ultrastructure of bone cells in Paget's disease of bone. J Bone Miner Res. 2006;21 (Suppl 2):P51-4.

3. Roodman GD. Studies in Paget's disease and their relevance to oncology. Semin Oncol. 2001;28(Suppl 11):15-21.

4. van Staa TP, Selby P, Leufkens HG, Lyles K, Sprafka JM, Cooper C. Incidence and natural history of Paget's disease of bone in England and Wales. J Bone Miner Res. 2002;17:465-71. 
5. Mangham DC, Davie MW, Grimer RJ. Sarcoma arising in Paget's disease of bone: declining incidence and increasing age at presentation. Bone. 2009;44:431-6.

6. Hansen MF, Seton M, Merchant A. Osteosarcoma in Paget's disease of bone. J Bone Miner Res. 2006;21(Suppl 2):P58-63.

7. Mankin HJ, Hornicek FJ. Paget's sarcoma: a historical and outcome review. Clin Orthop Relat Res. 2005;438:97-102.

8. Longhi A, Errani C, Gonzales-Arabio D, Ferrari C, Mercuri M. Osteosarcoma in patients older than 65 years. J Clin Oncol. 2008; 26:5368-73

9. Seton M. Paget disease of bone: diagnosis and drug therapy. Cleve Clin J Med. 2013;80:452-62.

10. Cowan RW, Singh G. Giant cell tumor of bone: a basic science perspective. Bone. 2013;52:238-46.

11. Chakarun CJ, Forrester DM, Gottsegen CJ, Patel DB, White EA. Matcuk GR Jr. Giant cell tumor of bone: review, mimics, and new developments in treatment. Radiographics. 2013;33:197-211.

12. Raskin KA, Schwab JH, Mankin HJ, Springfield DS, Hornicek FJ. Giant cell tumor of bone. J Am Acad Orthop Surg. 2013;21:118-26.

13. Viswanathan $\mathrm{S}$, Jambhekar NA. Metastatic giant cell tumor of bone: are there associated factors and best treatment modalities? Clin Orthop Relat Res. 2010;468:827-33.

14. Gianfrancesco F, Rendina D, Merlotti D, et al. Giant cell tumor occurring in familial Paget's disease of bone: report of clinical characteristics and linkage analysis of a large pedigree. J Bone Miner Res. 2013;28:341-50.

15. Jaffe HL. Tumors and tumorous conditions of bones and joints Philadelphia, PA: Lea and Fehiger; 1958.

16. Szendröi M. Giant-cell tumour of bone. J Bone Joint Surg Br. 2004;86:5-12.

17. Werner M. Giant cell tumour of bone: morphological, biological and histogenetical aspects. Int Orthop. 2006;30:484-9.

18. Shankman S, Greenspan A, Klein MJ, Lewis MM. Giant cell tumor of the ischium. A report of two cases and review of the literature. Skeletal Radiol. 1988;17:46-51.

19. Murphey MD, Nomikos GC, Flemming DJ, Gannon FH, Temple HT, Kransdorf MJ. From the archives of AFIP. Imaging of giant cell tumo and giant cell reparative granuloma of bone: radiologic-pathologic correlation. Radiographics. 2001;21:1283-309.

20. Dhillon MS, Prasad P. Multicentric giant cell tumour of bone. Acta Orthop Belg. 2007;73:289-99.

21. Hoch B, Inwards C, Sundaram M, Rosenberg AE. Multicentric giant cell tumor of bone. Clinicopathologic analysis of thirty cases. J Bone Joint Surg Am. 2006;88:1998-2008.

22. Dahlin DC. Caldwell Lecture. Giant cell tumor of bone: highlights of 407 cases. Am J Roentgenol. 1985;144:955-60.

23. Wang J, Pei F, Tu C, Zhang H, Qiu X. Serum bone turnover markers in patients with primary bone tumors. Oncology. 2007;72:338-42.

24. McKenna RJ, Schwinn CP, Soong KY, Higinbotham NL. Osteogenic sarcoma arising in Paget's disease. Cancer. 1964;17:42-66.

25. Wu RK, Trumble TE, Ruwe PA. Familial incidence of Paget's disease and secondary osteogenic sarcoma: a report of three cases from a single family. Clin Orthop. 1991;265:306-9.

26. Rendina D, Gianfrancesco F, De Filippo G, et al. Epidemiological, clinical, and genetic characteristics of Paget's disease of bone in a rural area of Calabria, Southern Italy. J Endocrinol Invest. 2010;33: 519-25.

27. Rendina D, Gennari L, De Filippo G, et al. Evidence for increased clinical severity of familial and sporadic Paget's disease of bone in Campania, Southern Italy. J Bone Miner Res. 2006;21:1828-35.

28. Gennari L, Gianfrancesco F, Di Stefano M, et al. SQSTM1 gene analysis and gene-environment interaction in Paget's disease of bone. J Bone Miner Res. 2010;25:1375-84.

29. Zlotogora J. High frequencies of human genetic diseases: founder effect with genetic drift or selection? Am J Med Genet. 1994;49:10-3.

30. Gianfrancesco F, Rendina D, Di Stefano M, et al. A nonsynonymous TNFRSF11A variation increases NFKB activity and the severity of Paget's disease. J Bone Miner Res. 2012;27:443-52.

31. Albagha OM, Visconti MR, Alonso N, et al. Common susceptibility alleles and SQSTM1 mutations predict disease extent and severity in a multinational study of patients with Paget's disease. J Bone Miner Res. 2013;28:2338-46.

32. Albagha OM, Wani SE, Visconti MR, et al. Genome-wide association identifies three new susceptibility loci for Paget's disease of bone. Nat Genet. 2011;43:685-9. 